\title{
PENAMBAHAN ASAM OLEAT TERHADAP SISTEM TRANSPOR Cu(II) DENGAN ZAT PEMBAWA OKSIN MELALUI TEKNIK MEMBRAN CAIR FASA RUAH
}

\author{
Olly Norita Tetra, Zaharasmi dan Refinel \\ Laboratorium Kimia Fisika Jurusan Kimia FMIPA Universitas Andalas
}

\begin{abstract}
The removal of $\mathrm{Cu}$ (II) from aqueous solutions had been employed throught bulk liquid membrane techniques with arrange the optimization conditions of transport $\mathrm{Cu}(\mathrm{II})$ interface system. The optimum conditions of transport were found to be $3.15 \times 10^{-4} \mathrm{M}$ of $\mathrm{Cu}(\mathrm{II})$ at $\mathrm{pH} 3$ in the source phase, $17.5 \times 10^{-4} \mathrm{M}$ oxine dissolved into chloroform as membrane phase, $0,15 \mathrm{M} \mathrm{H}_{2} \mathrm{SO}_{4}$ as acceptor in receiving phase, stirring rate was $340 \mathrm{rpm}$ and it was found that the transport of $\mathrm{Cu}(\mathrm{II})$ to receiving phase reached $97.41 \%$ during 6 hours. The effect of addition oleat acid $1.57 \times 10^{-3} \mathrm{M}$ as surfactant in membrane phase resulted a rapidly time of transport $\mathrm{Cu}$ (II) to be 3 hours, wherein $\mathrm{Cu}$ (II) transported into the receive phase reached $97.83 \%$ and remained in feed phase $0 \%$.
\end{abstract}

Keywords: bulk liquid membrane, oxine, oleat acid, Cu(II) )transport

\section{DAFTAR PUSTAKA}

1. M. Mulder, Basic Principle of Membrane Technology, Kluwer Academic Publisher, Dordrecht, 1991, 244 - 259.

2 H. Alpoguz, Ahmed Kaya, Mehmed Karakus, Mechanism and Kinetics of Copper(II) Transport Through a Liquid Membrane Containing a Dithio phosphonate Derivative as Carrier, Turk. J. Chem., $29:$ 345-353, (2005).

3. F. Khalil, and M. Shamsipur, Separation Study of Cadmium as $\mathrm{CdI}_{4}{ }^{2-}$ Through a Bulk Liquid Membrane Containing Ketoconazole and Oleaic Acid, Anal Sci., 21: 501-505, (2005).

4. G. Leon, Facilitated Transport of Cobalt Through Bulk Liquid Membranes Containg Diethylhexyl Phosphoric Acid, Desalination, 162:211-215, (2004).

5. M. D. Granado-Castro, M. D. GalindoRiano, M. Garcia-Vargas, Model Eksperiments To Test The Use Of A Liquid Membrane For Separation And Preconcentration Of Copper From Natural Water, Anal. Chim. Acta, 506: 81-86, (2004).

6. H. Korkmaz, Ahmet and Mehmet, Mechanism and Kinetics of $\mathrm{Cu}$ (II) Transport Throught a Liquid Membrane
Containing a Dithiophosphonate Derivative as Carrier, Turk. J. Chem., 29 : 345-353, (2005).

7. Z. Kahar, Mempelajari Peranan Oksin Sebagai Zat Pembawa Co(II) Antar Fasa (air-kloroform-air) Melalui Teknik Membran Cair Fasa Ruah, J. Kimia Andalas, 8(2), 29-33, (2002).

8. Z. Kahar, A. Alif, H. Aziz, dan Emriadi, Pengaruh ion $\mathrm{Fe}(\mathrm{III}), \mathrm{Ni}(\mathrm{II}), \mathrm{Cu}(\mathrm{II})$ Terhadap Transpor Co(II) Antar Fasa (airkloroform-air) Melalui Teknik Membran Cair Fasa Ruah, J. Kimia Andalas, 8(1), 29-33, (2002).

9. Z. Kahar, Mempelajari Transpor Cd(II) Antar Fasa Untuk Teknik Pemisahan Melalui Membran Cair Fasa Ruah Dengan Menggunakan Oksin Sebagai Zat Pembawa, Laporan Penelitian Project TPSDP Research Grant Jurusan Kimia FMIPA Unand, 2005.

10. O. Norita, Transpor Antar Fasa dari Ion Tembaga (II) Melalui Membran Cair Fasa Ruah. Thesis Program Pascasarjana, Universitas Andalas, Padang, 2001.

11. Shirtin, Kinetika Transpor $\mathrm{Cu}(\mathrm{II})$ dengan Zat Pembawa Oksin dan Asam Oleat Sebagai Zat Aditif Melalui Teknik Membran Cair Fasa Ruah, Skripsi, Jurusan Kimia, Universitas Andalas, Padang, 2006. 
12. Dean, Langer's Handbook of Chemistry Ed $13^{\text {th }}$. New York. 1985, 88-89.
13. Mellan, Organic Reagents in Anorganic Analysis. Wiley Erterm Limited, 1982, 31108. 